\title{
Dynamic Freedom: Substrate Stress Relaxation Stimulates Cell Responses
}

\author{
Kamol Dey ${ }^{1,2 *}$, Silvia Agnelli ${ }^{1}$ and Luciana Sartore ${ }^{1}$ \\ ${ }^{1}$ Department of Mechanical and Industrial Engineering, Italy \\ ${ }^{2}$ Department of Applied Chemistry and Chemical Engineering, Faculty of Science, Bangladesh \\ *Corresponding author: Kamol Dey, Department of Mechanical and Industrial Engineering, Italy
}

Submission: 侮 September 02, 2018; Published: 監 November 27, 2018

\section{Mini Review}

Natural tissues comprise a population of cells, extra cellular matrix (ECM) and signaling biomolecules. The only living component, cell, interacts with each other and with the ECM in an incredibly multifaceted way. From the materials point of view, cells are highly dynamic viscoelastic materials; reside in highly dynamic three dimensional (3D) viscoelastic ECM, communicate with its surrounding in a highly dynamic and complex way, and form a highly dynamic viscoelastic tissue [1,2]. The cells can continually read the microenviromental cues such as stiffness, porosity, topography etc. and respond to them in a mechano-sensitive way to maintain the both cellular and tissue health [3]. It has been long recognized that matrix integrity or stiffness has profound influences on cell function and fate [4-7]. Furthermore, cells exert forces on the matrix and gauge the feedback to make cell-fate decisions [8]. In a recent study, Chaudhuri et al. [9] show that stress relaxation, the ability of the matrix to dissipate cell-exerted forces, is a key mechanical signal to modulate stem cells fate and activity [9]. They tuned the stress relaxation response of the alginate hydrogels by altering alginate architecture using low to medium to high molecular weight alginate in combination with different crosslinking densities of calcium and covalent coupling of short polyethylene glycol as spacer to the gels, while keeping nearly constant initial elastic modulus and degradation, with an aim to decouple the initial elastic modulus and degradation from stress relaxation rate, and found that gels with faster stress relaxation enhanced cell spreading proliferation, and osteogenic differentiation of mesenchymal stem cells (MSCs), and further showed that these effects of stress relaxation were mediated by adhesion-ligand binding, actomyosin contractility and mechanical clustering of adhesion ligands. These findings support a model illustrated in Figure 1. Cells within a 3D matrix exert forces on the surrounding. In an elastic matrix, these cellular forces are stored within the static network indicating that there is no remodeling of the matrix microenvironment.

In contrast, in a viscoelastic matrix, theses forces can be relaxed over time through mechanical yielding and matrix remodeling. The stress relaxation rate is correlated with the degree of matrix remodeling: faster relaxation, greater remodeling. Stress relaxation is a dynamic mechanical cue that guides cells to mechanically remod el the matrix. In a fast-relaxing matrix, increased matrix remodeling facilitates the cell spreading, proliferation and differentiation.

In another study, Darnell et al. [10] examined whether stress relaxation rate can regulate bone formation in vivo by implanting alginate hydrogels with different rates of stress relaxation containing human MSCs into rat calvarial defects [10]. The authors found that the rats that received fast-relaxing gels showed significantly more new bone growth after three months than those that received slow-relaxing, stiffness-matched hydrogels. These two studies demonstrated that substrate stress relaxation can be a potential design parameter for promoting bone regeneration. Another recent study by Bauer et al. [11] revealed that spreading and proliferation of mouse myoblasts was greater in the soft stress-relaxing hydrogels compared to purely elastic hydrogels of the same initial elastic modulus [11]. In addition, Lee et al. [12] showed that faster stress relaxation of hydrogels promoted increased cartilage matrix production along with a wider volume of interconnected cartilage matrix [12]. In a very recent study, Ze Gong et al. [13] systematically examined the dynamics of motor clutches (i.e., focal adhesions) formed between the cell and a viscoelastic substrate using analytical methods and direct Monte Carlo simulation with an aim to decipher the mechanism of mechanosensitivity towards viscoelasticity of substrate [13]. Using both theoretical and experimental methods, they found that substrate viscosity showed no influence on cell spreading on the stiff substrates, while intermediate viscosity maximizes cell spreading on the soft substrates.

Taken together, these results highlight the importance of considering the matrix stress relaxation as a design parameter for materials in the tissue engineering. However, a time-resolved study is needed to fully elucidate the complex interdependence of cells and substrate stress relaxation kinetic that mediates optimal cell spreading in response to optimal stress relaxation rate similar to observing spatiotemporal relationship between the dynamics of focal adhesion systems and cell migration $[14,15]$. Finally, how do cells decide which response to trigger following a stress relaxation? It is clear that we are just only starting to understand and decipher how biological cells react to mechanical cues and further studies will enable discovery of all involved mechanisms fundamental in tissue growth and development. 


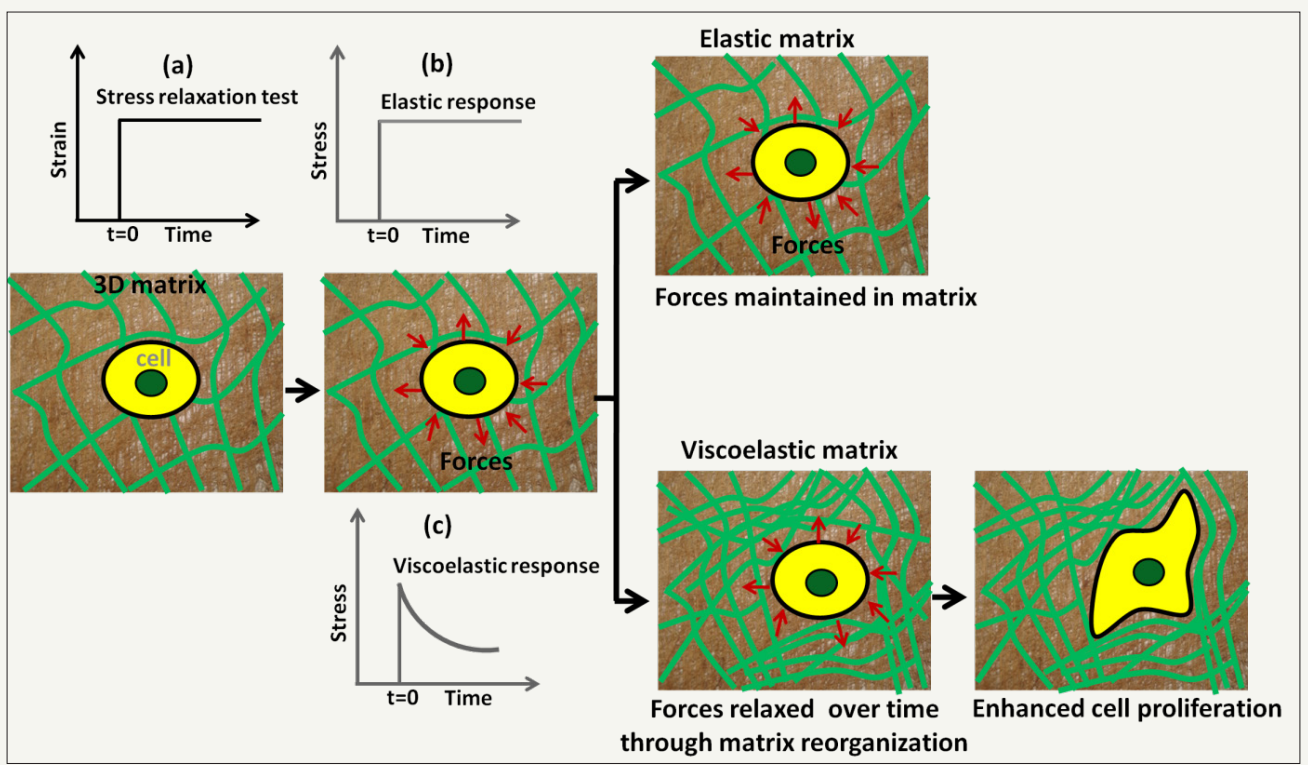

Figure 1: Hypothesis for how matrix mechanical cues modulate cellular behavior. An elastic matrix stores the cell-induced forces, while, a viscoelastic matrix dissipates these forces through matrix reorganization and facilitates cell proliferation and differentiation.

(a) Schematic representation of a stress-relaxation test. A strain is suddenly applied to the sample and subsequently kept constant, while the stress is recorded as a function of time.

(b) A static stress is observed for elastic sample (purely) whereas

(c) A dynamic stress, decreasing over time, is observed for a viscoelastic sample.

\section{References}

1. Kasza KE, Amy CR, Jiayu L, Thomas EA, Clifford PB, et al. (2007) The cell as a material. Curr opin cell biol 19(1): 101-107.

2. Hoffman BD, Carsten G, Martin AS (2011) Dynamic molecular processes mediate cellular mechanotransduction. Nature 475(7356): 316.

3. Humphrey JD, Eric RD, Martin AS (2014) Mechanotransduction and extracellular matrix homeostasis. Nature reviews Molecular cell biology 15(12): 802.

4. Engler AJ, Shamik S, Sweeney HL, Dennis ED (2006) Matrix elasticity directs stem cell lineage specification. Cell 126(4): 677-689.

5. Murphy WL, Todd CD, Adam JE (2014) Materials as stem cell regulators. Nature materials 13(6): 547-557.

6. Guo W, Margo TF, Nancy AB, Yu-li W (2006) Substrate rigidity regulates the formation and maintenance of tissues. Biophysical journal 90(6): 2213-2220.

7. Ulrich TA, Elena MP, Sanjay K (2009) The mechanical rigidity of the extracellular matrix regulates the structure, motility, and proliferation of glioma cells. Cancer research 69(10): 4167-4174.

8. Trappmann B, Julien E, John TC, Daniel GS, Yuan Li, et al. (2012) Extracellular-matrix tethering regulates stem-cell fate. Nature materials 11(7): 642-649.
9. Chaudhuri O, Luo G, Darinka K, Max D, Sidi AB, et al. (2016) Hydrogels with tunable stress relaxation regulate stem cell fate and activity. Nature materials 15(3): 326-334.

10. Darnell M, Simon Y, Luo G, Nisarg S, Evi L, et al. (2017) Substrate stressrelaxation regulates scaffold remodeling and bone formation in vivo. Advanced healthcare materials 6(1): 1601185.

11. Bauer A, Luo G, Brian K, Weiwei AL, Maxence D, et al. (2017) Hydrogel substrate stress-relaxation regulates the spreading and proliferation of mouse myoblasts. Acta biomaterialia 62: 82-90.

12. Lee HP, Luo G, David JM, Marc EL, Ovijit C (2017) Mechanical confinement regulates cartilage matrix formation by chondrocytes. Nature materials 16(12): 1243-1251.

13. Gong Z, Spencer ES, Steven RC, Elisabeth EC, Ovijit C, et al. (2018) Matching material and cellular timescales maximizes cell spreading on viscoelastic substrates. Proceedings of the National Academy of Sciences 115(12): E2686-E2695.

14. Hu YL, Shaoying L, Kai WS, Jie S, Yingxiao W, et al. (2014) FAK and paxillin dynamics at focal adhesions in the protrusions of migrating cells. Scientific reports 4: 6024

15. Gupton SL, Clare MW (2006) Spatiotemporal feedback between actomyosin and focal-adhesion systems optimizes rapid cell migration. Cell 125(7): 1361-1374. 
Creative Commons Attribution 4.0 International License

For possible submissions Click Here

Submit Article

RMES $\begin{gathered}\text { Researchin } \\ \text { Medical } \\ \text { Engineering } \\ \text { Sciences }\end{gathered}$
Benefits of Publishing with us
- High-level peer review and editorial services
- Freely accessible online immediately upon publication
- Authors retain the copyright to their work

